Key points

- Multidisciplinary evaluation is always preferred and all areas of disease involvement should be evaluated.

- Radiotherapy is a valuable treatment option for pain, bleeding, obstruction and symptoms due to mediastinal involvement.

- Typical palliative radiotherapy treatment courses take approximately $1-3$ weeks. Shorter-course treatments may often be discussed.

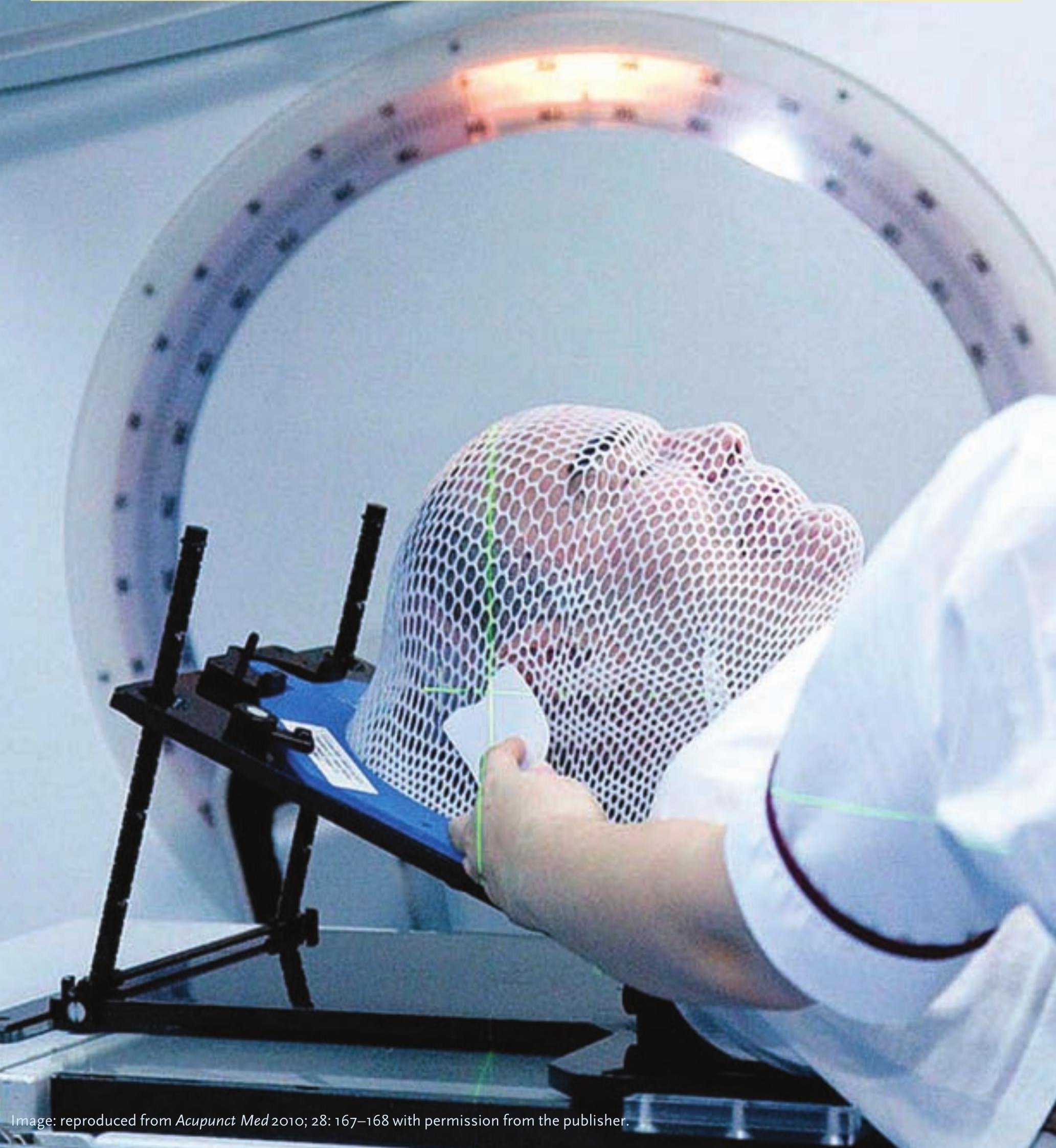




\begin{tabular}{lll}
\hline P.M. Putora' & 0 & D \\
M. Früh & 'Dept of Radiation & Dept of Radiation Oncology, paul.putora@kssg.ch \\
Oncology & Cantonal Hospital St Gallen \\
L. Kern & 'Dept of Haematology and & (Kantonsspital St. Gallen), \\
& Oncology & 9007 St. Gallen, Switzerland \\
& 'Dept of Pneumology, & \\
& Cantonal Hospital St Gallen \\
& (Kantonsspital St Gallen), \\
& St Gallen, Switzerland
\end{tabular}

\section{The place of radiotherapy in the palliative management of NSCLC}

\section{Educational aims}

$\odot$ To explain the basic rationale of radiation oncology.

$\odot$ To set out the typical indications for palliative radiotherapy and its limitations.

\section{Summary}

Palliative treatment may be needed for many patients with nonsmall cell lung cancer (NSCLC) at diagnosis or during the course of their disease. For many palliative problems, including bleeding, pain, superior vena cava syndrome and obstruction, there are various treatment options, with radiotherapy being one of them. Consequently, multidisciplinary evaluation before a treatment decision is made is very important.

\section{Clinical presentation}

At diagnosis, more than half of all patients with lung cancer are diagnosed with stage IV disease. Unfortunately, most patients diagnosed with lower stages subsequently progress, and require palliative treatment $[1,2]$.

Common clinical presentations include cough, haemoptysis, dyspnoea and pain (resulting from involvement of the pleura, chest wall or mediastinal structures), which usually indicates advanced disease. Some patients initially present with general symptoms such as weakness, anorexia, weight loss and malaise.

Some symptoms are connected to specific tumour locations:

- Superior sulcus tumours may cause Pancoast's syndrome (consisting of shoulder pain, brachial plexopathy and Horner's syndrome). Horner's syndrome is characterised by enophthalmus, ptosis and miosis.

- Lymphadenopathy of the aortopulmonary window and predominantly left lung tumours may cause through involvement of the recurrent laryngeal nerve.
Statement of interest None declared.
HERMES syllabus link: module B.2.1 
- Dyspnoea due to paralysis of the hemidiaphragm may be caused by phrenic nerve involvement.

- Dysphagia or even odynophagia may occur when the tumour or lymph nodes cause compression of the oesophagus.

- Involvement of the right mediastinum may cause superior vena cava syndrome.

In addition to local symptoms, paraneoplastic syndromes should be assessed carefully as they occur frequently, particularly in small cell lung cancer. Syndromes include the production of adrenocorticotropic hormone (ACTH) by the tumour, causing cortisol oversecretion by the adrenal glands (Cushing's syndrome) as well as increased parathormone levels, Syndrome of Inappropriate Antidiuretic Hormone Secretion (SIADH), Lambert Eaton syndrome and others.

There are various situations where radiotherapy can be a valuable treatment option. The most common sites of metastases are lung, liver, brain and bones.

\section{Multidisciplinary evaluation}

Palliative treatment focuses on alleviating symptoms and improving or stabilising quality of life. In patients requiring palliation, special focus needs to be directed towards weighing the side-effects and benefits of treatment. With different approaches available, multidisciplinary discussion is similarly important as it is in treatment with curative intent. Radiotherapy can be a very effective method, but should undergo careful interdisciplinary evaluation.

\section{Radiotherapy}

Radiotherapy is the use of ionising radiation in the treatment of cancer. Radiotherapy can be applied with linear accelerators (often known as linacs) using high-energy X-rays (or cobalt machines using gamma radiation). The rays may travel from the outside of the body to their target - this is termed external beam radiotherapy (EBRT) - or the radiation source may be placed endobronchially - this is an application of brachytherapy.

\section{Physics}

The linear accelerator accelerates electrons using microwave technology to almost light-speed. These electrons collide with a heavy metal target, producing high-energy $\mathrm{X}$-rays from the target (so-called Bremsstrahlung). These high-energy $X$ rays are shaped as they exit the machine to conform to the shape of the tumour, and the shaped beam is directed to the tumour.

The beam may be shaped either by blocks that are placed in the head of the machine or by a multileaf collimator incorporated into the head of the machine.

\section{Units}

In radiotherapy, the unit used for absorbed dose is the gray (Gy). One gray is defined as

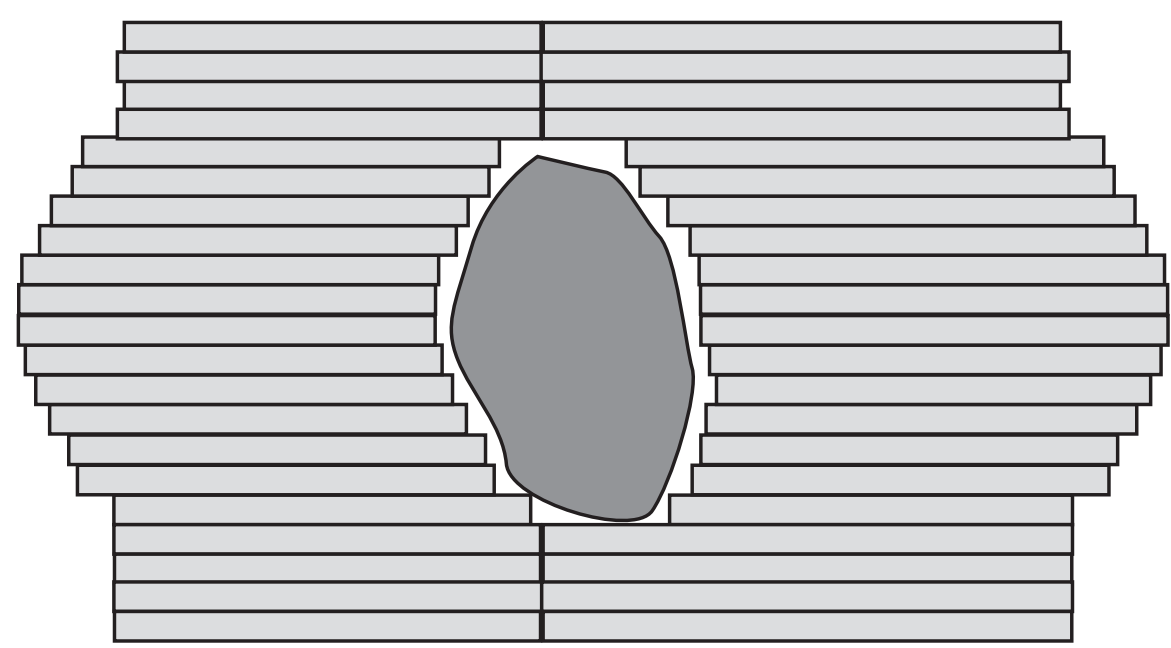

Figure 1

A multileaf collimator used to form the treatment beams. 
the absorption of one joule of ionising radiation by one kilogram of matter. An alternative unit that used in the past was the rad, which is equivalent to 0.01 Gy (also termed 1 cGy). Typically a dose of 2 Gy per day on 5 days per week is termed standard fractionation. Besides the physical dose, the biological effect also depends on the fractionation schedule as well as tissue properties.

\section{Radiobiology}

Lung cancers, like most other cancers, are made of cells that typically divide more rapidly than those in normal tissue. Radiotherapy acts by attacking the DNA of tumour cells, disabling their ability to survive and undergo further cell division. Typically, the same damage is dealt to the normal cells immediately surrounding the tumour cells. Typically, the radiotherapy dose is applied in daily fractions large enough to damage a high percentage of the rapidly dividing tumour cells, but small enough to allow most normal tissue cells to repair and survive.

As surviving cancer cell numbers decrease with increasing radiation dose, an extremely high dose of radiotherapy should ideally be applied. Unfortunately, increasing the dose of radiotherapy also means increasing the early and late side-effects of treatment. The optimal dose is found when a reasonable effect can be achieved with reasonable side-effects. In contrast to a typical curative treatment, where the aim is total local tumour eradication, tumour size reduction may be sufficient

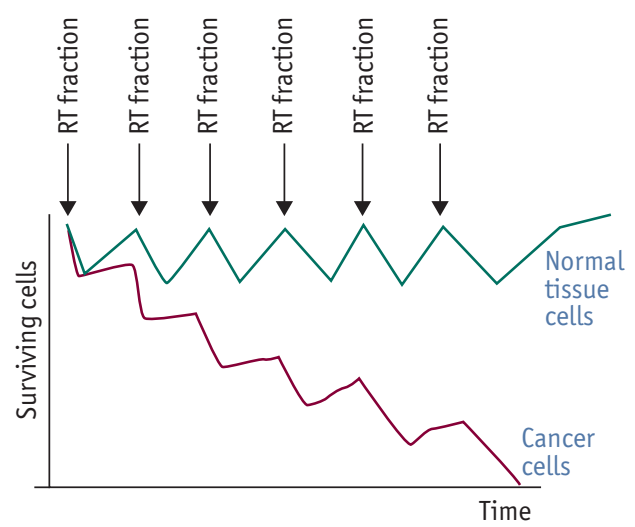

Figure 2

After fractionated radiotherapy, tumour cells are not capable of the same degree of repair as normal tissue cells. In the ideal situation, all tumour cells are eliminated while normal tissue is spared. in palliative situaitons. The threshold for sideeffects during palliative treatment is lower. This is why the typical doses for palliative treatment tend to be smaller than in the curative setting.

However, single higher-dose irradiation is often applied in palliative radiotherapy (e.g. 8 Gy per fraction; in contrast to typical fractionation regimes using 2-3 Gy per fraction). These higher doses per fraction are expected relatively to increase the late sideeffects. In most palliative therapies, late effects are often of less concern than they are in curative efforts.

Before a treatment can be started, the volume to be treated needs to be defined. This may be done directly via simulator (X-ray image) or by three-dimensional imaging with the help of a planning computed tomography (CT) scan.

Typically, the gross tumour volume (GTV) is contoured. This is the region where tumour is known to be present. The GTV is then expanded, typically by $5-10 \mathrm{~mm}$, to include microscopic involvement: this forms the clinical target volume (CTV). An additional margin may account for movement due to breathing or heartbeat: this is termed the intrafraction target volume (ITV). A last margin is applied to this volume, accounting for uncertainties in radiotherapy delivery and patient positioning: this is termed the planning target volume (PTV) [3].

To keep the target volume small and accurate, the patient must be in the same position during treatment as he/she was during imaging. To enable and verify the same patient, position markers (such as lines on the skin) are applied. In certain situations, immobilisation

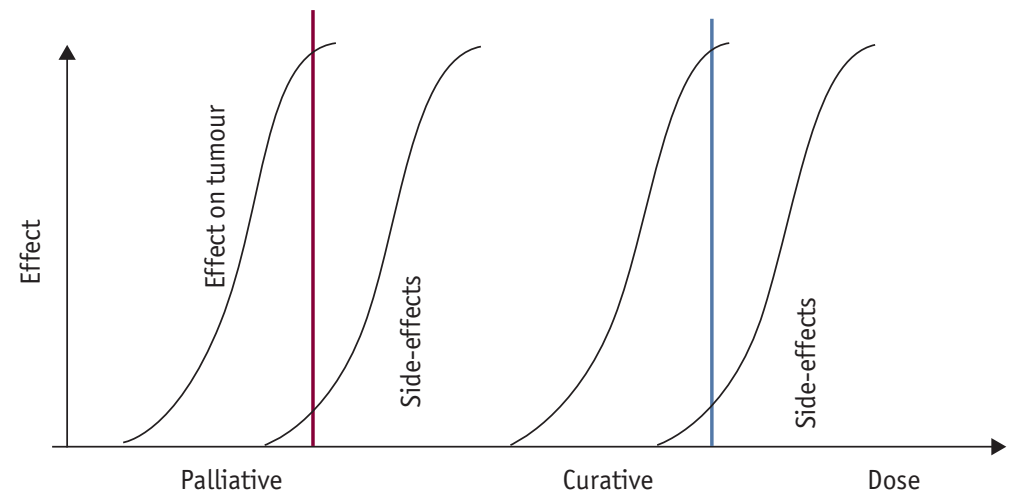

Figure 3

The optimal dose is that which causes maximal tumour damage while sparing normal tissue. Due to different goals in effect and a different willingness to risk side-effects, doses for palliative treatment are typically lower. 


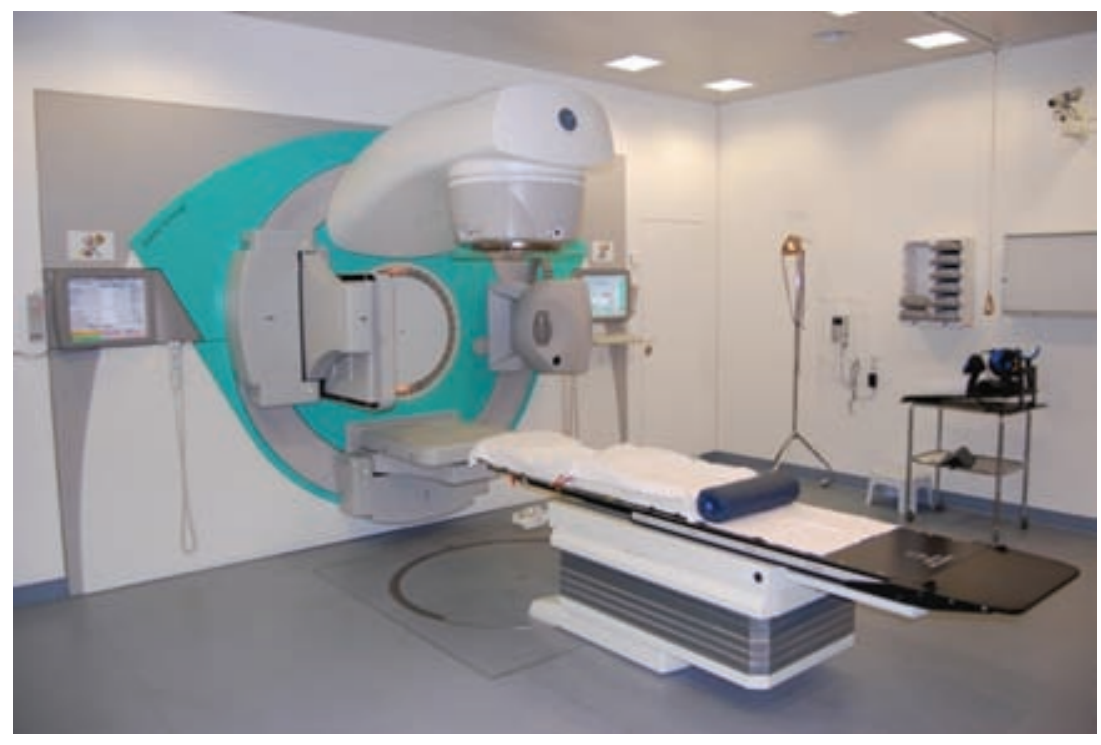

Figure 4

A linear accelerator. The gantry is shown above the table. Depending on the treatment plan, the gantry rotates around the table to deliver the planned dose.

devices are used to minimise patient movement (such as head masks for brain irradiation). The position markers for the treatment do not necessarily overlap with the treatment volume; this should be explained to patients.

The patient lies on a moveable treatment couch and lasers are used to make sure the patient is in the proper position. The treatment couch can move in many directions in all three planes. The radiation beam is emitted from the gantry of the accelerator. Radiation can be delivered to the tumour from any angle by rotating the gantry and moving the treatment couch.

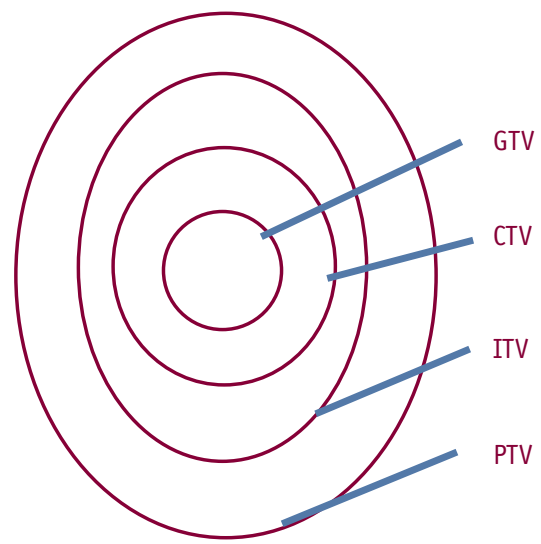

Figure 5

The defintion of the target volume by gross (GTV), clinical (CTV), intrafraction (ITV) and planning (PTV) target volume.

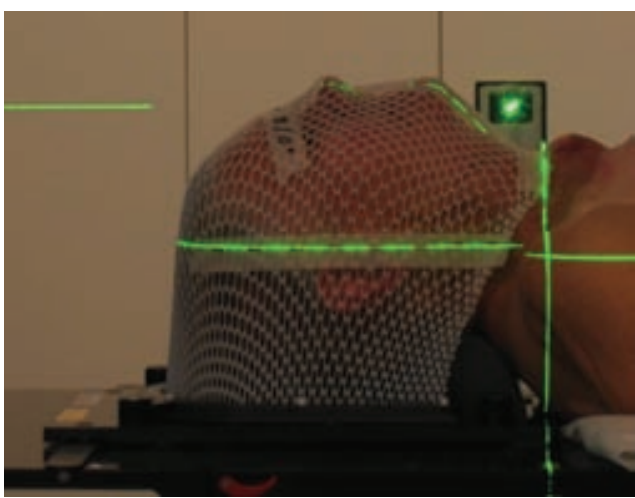

Figure 6

Mask for head immobilisation. Masks may also cover the shoulder region.

\section{Dose-volume histograms}

Radiation therapy planning involves the definition of the target volume as well as the organs at risk. Based on studies and previous analysis, there is a general understanding of the approximate doses each organ can receive without a relevant risk for higher-grade toxiticies (lungs, heart, spinal cord). Dose-volume histograms (DVHs) are plots from which the distribution of radiation dose to specific volumes can be displayed. These can be calculated automatically by modern treatment planning systems. In most palliative cases, the doses applied are in the lower ranges and usually do not represent a limiting factor in patients who have not been previously irradiated. On the other hand, patients with previous irradiation need to be

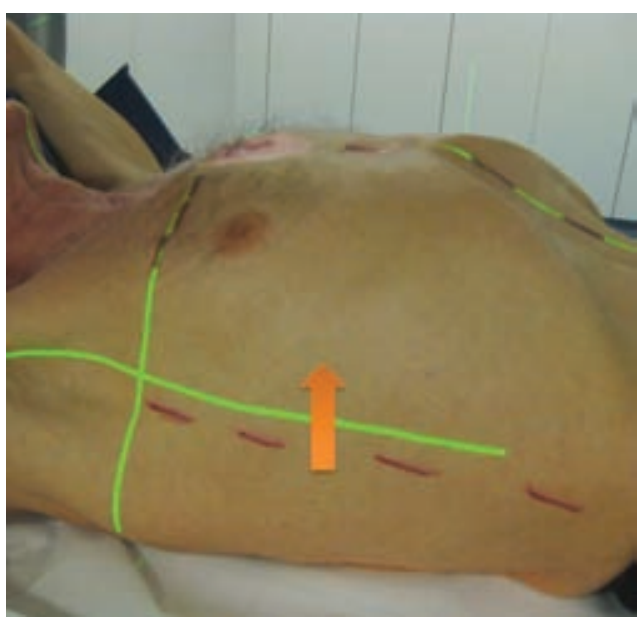

Figure 7

Lines as positions markers for external beam radiotherapy. The treatment couch is too low in this image. When it is moved up; the patient will be in the correct treatment position. 
carefully evaluated, as the risk of side-effects can increase significantly depending on the total dose and partially on the recovery time. Despite the lack of evidence-based data, diffusion capacity for carbon monoxide $(D L, C O)$ levels of $<40 \%$ predicted are generally considered to be a contraindication for curative radiotherapy. However, in palliative situations, $D \mathrm{~L}, \mathrm{CO}$ and vital capacity impairment are usually not limiting factors, since dose and treatment volume are relatively small.

\section{Timing issues and response}

\section{Pre-treatment}

Treatment with radiation therapy should not be paused for radiobiological reasons (this would allow tumour repopulation). The treatment preparation includes patient evaluation, written consent, a planning CT or simulation (direct X-ray planning), treatment planning, calculation, assessment and verification. Often, additional parameters, such as additional imaging or spirometry, are required before radiotherapy can start. For these reasons and due to interdependencies of these steps, several days are often required before treatment can start.

\section{Post-treatment}

For almost all radiation treatments, the response does not occur immediately. Typically the first response can be detected after several days. Improvement in symptoms, such as bone pain in metastasis, can take up to several weeks to fully respond. Due to the varying intensity of symptoms during radiotherapy, change of medication should be initiated only after a steady state has been achieved.

The timing of the treatment itself should be decided based on symptoms. In asymptomatic patients or in case of symptoms that are adequately controllable by other treatments, delaying radiotherapy is a favourable option.

In case of total atelectasis of the lung due to malignant bronchial obstruction, treatment should start within 2 weeks, as this has shown to provide better results.

\section{Retreatment}

Although radiotherapy is an effective therapy, recurrence of tumour or symptoms in the same or a nearby location may occur. Depending on previous dose, re-irradiation may be considered.

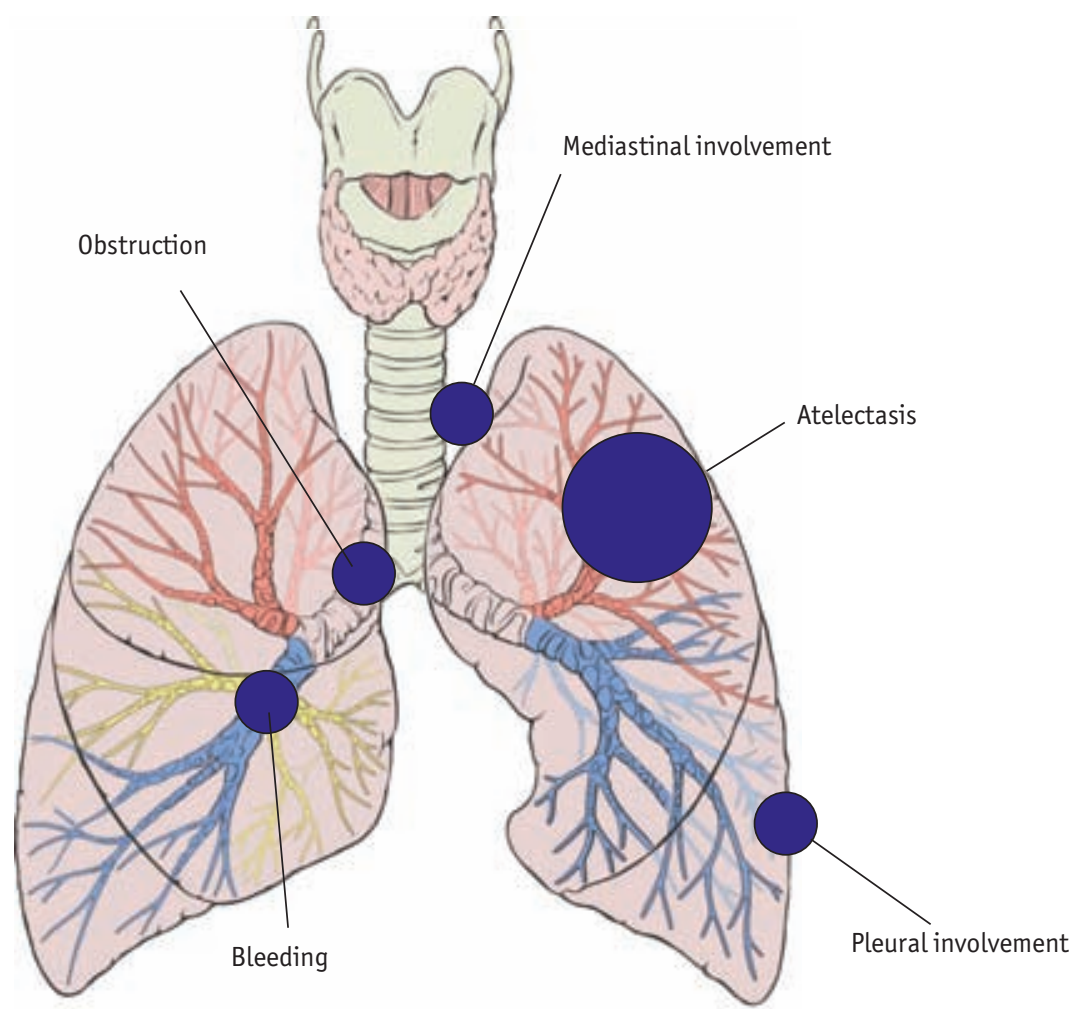

Figure 8

Lung cancers may cause pain, bleeding, atelectasis and mediastinal involvement with a variety of symptoms. Many of these can be treated succesfully with radiotherapy.

This is often limited in dose due to the prior strain on the normal tissues.

\section{Regimens}

Different regimens have been compared for palliation of thoracic symptoms such as bleeding, pain and obstruction. There is no clear evidence to favour one fractionation regimen over another. The options start with short-course therapies with $1 \times 10 \mathrm{~Gy}, 2 \times 8.5$ Gy one week apart. A very commonly used regime is $10 \times 3$ Gy $[4,5]$. In selected cases, higher doses may be considered (up to $>40 \mathrm{~Gy}$ ). There seems to be a trend towards better overall survival with higher dose regimens in patients with better performance status. There has been no difference in time to treatment response or effectiveness. The possible advantage of the longer regimens needs to be weighed against the longer treatment times ( 1 or 2 days for shortcourse regimes compared to over 4 weeks for the high-dose regimes). There also seems to be a small risk for myelopathies with the shortcourse therapies, although this risk can mostly be avoided with three-dimensional treatment planning and delivery. 


\section{Indications for palliative radiotherapy}

\section{Obstruction and atelectasis}

When dealing with endobronchial or endotracheal tumours, the primary task is to secure the airways in order to prevent complete obstruction which leads to post-stenotic pneumonia and atelectasis of the whole lung or one or more lobes. Endoscopic procedures using flexible or rigid bronchoscopy are the initial treatment of choice in these situations. A broad range of expandable stents is available to secure airway patency. In many cases, partial excision of the endoluminal tumour is necessary using YAG laser, argon plasma coagulation or cryotherapy. Radiotherapy can also be used as a primary treatment in selected cases or as an adjuvant treatment after an endoscopic procedure. Usually, EBRT is applied and, in selected cases, an additional boost with brachytherapy may be evaluated. Although the idea of brachytherapy is very appealing since the treatment is applied directly to the tumour, there are no clear data for improved outcome in patients treated with this modality. Also, the recently published guidelines of the American Society for Radiation Oncology state that there is currently no clear evidence that brachytherapy should be recommended alone or in combination with EBRT [5]. However, brachytherapy may play a role in selected cases, depending on local experience and availability.

\section{Pain management}

Tumours infiltrating the pleura or mediastinum can cause severe pain. Radiotherapy represents a very effective treatment modality for this indication. Once the location of the infiltration is defined, usually on CT scans, this region is delineated by the radiation oncologist. Treatment is usually performed with EBRT in several sessions depending on the performance status of the patient and his ability to lie still on the treatment couch. For palliative radiotherapy for bone pain, several regimes are used, including $1 \times 8 \mathrm{~Gy}, 2 \times 8 \mathrm{~Gy}$, $3 \times 6 \mathrm{~Gy}, 5 \times 4 \mathrm{~Gy}, 10 \times 3 \mathrm{~Gy}$ and $20 \times 2 \mathrm{~Gy}$. The regimen applied often depends on the experience of the individual centre.

In about one patient in five, radiotherapy may cause a temporary swelling in the region of infiltration, causing the pain to increase. This can partially be counteracted by systemic steroid treatment, although the evidence for this is not yet clear. It is of central importance that patients and physicians alike understand that the effect of radiotherapy becomes noticeable only after a reduction in tumour infiltration or reduction in tumour mass, as this effect leads to decreased pressure. Therefore, patients need to have sufficient analgesic medication to bridge the time gap until radiotherapy causes pain relief. In a few cases, this may take up to a several weeks. The physician should not forget to inform the patient that symptoms may worsen temporarily for a few days after the initiation of treatment.

\section{Superior vena cava syndrome}

Due to tumour extension, tumour infiltration or lymph node mass effects, the superior vena cava may be obstructed causing a decreased or even interrupted blood flow from the upper body. Symptoms include swelling of the neck and arms as well as facial plethora. Initial treatment depends on the severity of the symptoms and the tumour histology. While in small cell lung cancer or lymphoma, prompt initiation of chemotherapy may rapidly alleviate symptoms, patients with NSCLC are best treated with radiotherapy and/or stenting. The role of steroids is controversial in patients with NSCLC histology.

\section{Bleeding}

Haemoptysis is a severe and potentially dangerous complication of intrabronchial tumours. Since the total volume of the bronchi is not greater than about $150 \mathrm{~mL}$, patients with severe haemoptysis in acute danger of death from asphyxia. Furthermore, coughing up blood is enormously frightening for many patients, so the psychological effects of haemoptysis should not be underestimated. There is no evidencebased consensus about diagnostics in acute haemoptysis. In case of severe haemoptysis or in patients without previously known tumorous disease [4], an initial approach with bronchoscopy is certainly worthwhile as it can lead to the identification of the bleeding site in case of a tumour in the central airways, it can be used to aspirate blood and - importantly - it is helpful to guide selective intubation to secure ventilation of the noninvolved side in case of massive haemoptysis and imminent asphyxia. 
Intrabronchial approaches may be used to still bleeding by direct intervention such as YAG laser, argon plasma coagulation and/or stenting. In case of a bleeding site beyond the reach of the bronchoscope, a CT scan is helpful to precisely define the source. In some situations such as diffuse bleeding or bleeding from locations beyond the reach for intrabronchial interventions, radiotherapy represents a valuable option. Target definition is based on radiological imaging and endobronchial findings if it is possible to determine the source of bleeding. The regimes used for bleeding maybe include single-fraction treatments or fractionated treatments.

However, in acute situations without the possibility of a bronchoscopic intervention, patients must be stabilised and bleeding must be controlled by conservative means including inhalation with adrenaline or terlipressin, intubation and cough suppression.

\section{Pleural effusion}

Although pleural effusion is a common problem in lung cancer patients, radiotherapy does not represent a treatment option for this condition. In recurring pleural effusion, the mainstay of treatment is talc pleurodesis. Alternatively, pleurodesis can be performed using bleomycin or vibramycin.

\section{Combined therapy}

Although the response rate might be slightly increased with the combination of chemotherapy and radiation, radio-chemotherapy is generally not recommended in the palliative setting as it typically increases the side-effects of treatment. The combination of radiotherapy and targeted therapy in the palliative setting has not yet been sufficiently explored and is currently not standard care.

\section{Side-effects of thoracic radiotherapy}

Acute effects

During radiotherapy, many patients describe fatigue; in some cases this may be so strong that it limits them in their daily activity. As most patients are additionally burdened by having to travel to the hospital daily, change of sleeping pattern, loss of appetite and psychological stress, these issues need to be addressed. Typically, fatigue is strongest towards the end of treatment and requires several days or even weeks to improve. Physical activity does not need to be limited during radiotherapy, though patients are entitled to reduce their workload and rest if they prefer to.

Depending on the location of radiotherapy and the dose applied, most patients should expect hair loss in irradiated regions. Typically, hair loss on the chest is not a very disturbing side-effect. Patients need to be informed to expect hair loss several weeks after treatment, though most can expect hair to regrow later.

Erythema of the skin is a rather common side-effect. In typical palliative dose regimens, symptoms are rather rare. In case of symptoms due to irritation by radiation, they include dry skin, erythema, itchiness and tenderness. Desquamation is extremely rare in palliative doses. As long as patients show no symptoms, no treatment is required. In case of skin irritation, additional strong sunlight exposure should be avoided or sunscreen used.

If the treated target is in the mediastinum, oesophagitis may occur. Sometimes this may cause a feeling of a foreign mass, dysphagia or even odynophagia. These symptoms typically resolve within a few weeks. Due to radiotherapy and often a compromised immune system (often a side-effect of previous chemotherapy) oesophagitis should always be thought of and treated if suspected.

\section{Late effects}

Radiation pneumonitis may develop after approximately 10 weeks after radiotherapy, though this is not typically seen with palliative doses. Symptoms include coughing and shortness of breath and sometimes fever. It can be diagnosed by radiological imaging where the main pattern of inflammation follows the original treatment field arrangements. If required, the treatment of choice is cortisone.

\section{Follow-up}

As palliative treatment focuses on the symptoms of the patient, the best way to judge the success of treatment is the clinical presentation of the symptoms. For pain, dysphagia or dyspnoea, the effect of treatment can be best judged by patients themselves. 


\section{Indications for extrathoracic palliative radiotherapy}

\section{Brain metastasis}

Brain metastases are a very common symptom of NSCLC. Radiotherapy is the primary treatment of choice. Due to the lower efficacy of systemic chemotherapy "above" the bloodbrain barrier, brain metastases present a large common problem, especially in advanced NSCLC. Fractionated radiotherapy of the whole brain is usually performed. Regimes most commonly used range from $5 \times 4$ Gy to $10 \times 3$ Gy [6]. Due to the low life expectancy of patients with brain metastasis, possible late side-effects of shorter regimes such as possible concentration problems might be compensated by a reduced number of fractions, meaning fewer treatment days. The acute side-effects do not vary significantly and may include nausea, headaches, fatigue, loss of appetite and always hair loss. Nausea and headaches may be counteracted with cortisone treatment during whole-brain radiotherapy and with dexamethasone $3 \times 4 \mathrm{mg}$ per day and then tapered off once radiotherapy is finished.

Symptoms obviously depend on the location and extent of the lesions. Predicting the devolution of symptoms is practically impossible. In some patients, it may take several weeks until symptoms are improved.

\section{Multidisciplinary note}

In addition to whole-brain radiotherapy, patients with singular metastasis should be evaluated for either neurosurgery or stereotactic radiotherapy (intensive focal radiotherapy), which should be discussed with less than four brain metastases. In patients with epidermal growth factor receptor (EGFR) mutation, up-front tyrosine kinase inhibitors lead to high response rates in brain metastases and whole-brain irradiation may be delayed when patients are asymptomatic.

\section{Side-effects}

Symptoms that may occur during radiotherapy include nausea, headaches and tiredness. Several weeks after treatment, hair loss develops: this is usually only temporary. Problems with concentration and memory have been described, though the impact of disease and other treatments (e.g. chemotherapy) should not be underestimated.

\section{Contraindications/caveats}

The performance status of the patient must always be critically evaluated. If the Karnofsky Performance Status is below 60\%, the indication needs to be critically judged.

\section{Bone metastasis}

Bone metastasis may be associated with pain, risk of fracture and neurological symptoms. Typical regimes used in radiotherapy for bone metastasis can be $5 \times 4$ Gy or $10 \times 3$ Gy. In selected patients, especially in patients without soft tissue involvement, $1 \times 8$ Gy can be a very good choice.

\section{Multidisciplinary note}

In very diffuse bone metastasis, radionuclide therapy, e.g. samarium therapy, should be evaluated and may rarely be a treatment option.

In particular, bone metastasis of weightcarrying bones should be evaluated by an orthopaedic surgeon. The systemic use of bisphosphates has shown to delay the onset of skeletal-related events.

\section{Side-effects}

It is very important to inform patients that bone pain might increase as a temporary side-effect of radiotherapy for a few days due to swelling. Otherwise, patients might consider this treatment failure or tumour progression. Side-effects on normal tissues depend on the location of the bone metastasis, as this influences normal tissue irradiation. With typical palliative doses, these side-effects are usually well tolerated.

\section{Contraindications/caveats}

Typically, concurrent chemotherapy should be paused if plausible. Bone marrow ablation may occur when extensive regions of blood-generating bones are irradiated; this needs to be taken into account as haematopoiesis is often limited due to pre-treatments in these patients.

It is recommended always to fully assess patients for possible indication of radiotherapy of multiple disease locations. Patients presenting with brain metastases may also have painful bony metastases at the same time. Thus, treatment of different locations is preferably carried out during the same session, decreasing the overall treatment time for the patient. 


\section{Further reading}

Rodrigues G, Videtic GMM, Sur R, et al. Palliative thoracic radiotherapy in lung cancer: an American Society for Radiation Oncology evidence-based clinical practice guideline. Pract Radiat Oncol 2011; 1: 60-71.

Spiro SG, Huber RM, Janes SM, eds. Thoracic Malignancies. Eur Respir Mon 2009; 44.

\section{References}

1. Hatton MQF, Martin J. Radiotherapy in the treatment of nonsmall cell lung cancer. In: Spiro SG, Huber RM, Janes SM, Thoracic Malignancies. Eur Respir Mon 2009; 44: 207-243.

2. Aurora R, Milite F, Vander Els NJ. Respiratory emergencies. Semin Oncol 2000; 27: 256-269.

3. International Commissionon of Radiation Units and Measurements. Prescribing, Recording and Reporting Photon Beam Therapy (Supplement to ICRU Report 50), ICRU Report 62. Bethesda, ICRU, 1999.
4. Lester JF, MacBeth F, Toy E, et al. Palliative radiotherapy regimens for non-small cell lung cancer. Cochrane Database Syst Rev 2006; 4: CDoo2143.

5. Rodrigues G, Videtic GMM, Sur R, et al. Palliative thoracic radiotherapy in lung cancer: an American Society for Radiation Oncology evidence-based clinical practice guideline. Pract Radiat Oncol 2011; 1: 60-71.

6. Soffietti R, Cornu P, Delattre JY, et al. EFNS Guidelines on diagnosis and treatment of brain metastases: report of an EFNS Task Force. Eur J Neurol 2006; 13: 674-681. 